\title{
On the Solution of Revenue- and Network-Constrained Day-Ahead Market Clearing under Marginal Pricing-Part I: An Exact Bilevel Programming Approach
}

\author{
Ricardo Fernández-Blanco, Member, IEEE, José M. Arroyo, Senior Member, IEEE, and Natalia Alguacil, Senior \\ Member, IEEE
}

\begin{abstract}
The first of this two-paper series addresses a practical day-ahead auction model where generation revenue constraints are explicitly incorporated in the problem formulation, as routinely done in several national electricity markets across Europe. The revenue-constrained market-clearing procedure includes the effect of the transmission network, inter-temporal constraints associated with generation scheduling, demand-side bidding, and marginal pricing. This auction design is an instance of price-based market clearing which features two major complicating factors. First, locational marginal prices become decision variables of the optimization process. In addition, producer revenues are formulated as bilinear and highly nonconvex products of power outputs and market-clearing prices. The resulting problem is formulated as a mixed-integer nonlinear bilevel program with bilinear terms for which available solution techniques rely on heuristics, approximations, or modeling simplifications. This paper presents a novel and exact methodology whereby the original problem is recast as an equivalent singlelevel mixed-integer linear program. As a consequence, finite convergence to optimality is guaranteed and the use of standard commercial software is allowed. The proposed transformation is based on duality theory of linear programming, Karush-KuhnTucker optimality conditions, and integer algebra results. In the second part of this two-paper series, numerical results from several case studies illustrate the effective performance of the proposed solution approach.
\end{abstract}

Index Terms-Bilevel programming, locational marginal pricing, mixed-integer linear equivalent, pool-based electricity market, price-based market clearing, revenue-constrained auction design.

\section{NOMENCLATURE}

For quick reference, the symbols used throughout this paper and its companion [1] are listed below.

\section{A. Indices}

$b \quad$ Demand bid block index.

This work was supported in part by the Ministry of Science of Spain, under CICYT Project ENE2015-63879-R, and the Junta de Comunidades de Castilla-La Mancha, under Project POII-2014-012-P.

Ricardo Fernández-Blanco is with the Department of Electrical Engineering, University of Washington, Seattle, WA 98195-2500, USA (e-mail: rfbc85@uw.edu).

José M. Arroyo and Natalia Alguacil are with the Departamento de Ingeniería Eléctrica, Electrónica, Automática y Comunicaciones, E.T.S.I. Industriales, Universidad de Castilla-La Mancha, Ciudad Real E-13071, Spain (e-mail: JoseManuel.Arroyo@uclm.es; Natalia.Alguacil@uclm.es ).

$\begin{array}{ll}h & \text { Time period index. } \\ i & \text { Generating unit index. } \\ j & \text { Consumer index. } \\ l & \text { Transmission line index. } \\ n & \text { Bus index. } \\ o & \text { Generation offer block index. } \\ t & \text { Time period index. }\end{array}$

B. Sets

$\mathcal{B}_{j} \quad$ Index set of the demand bid blocks of consumer $j$.

$\mathcal{F}_{i t} \quad$ Feasibility set for the scheduling variables associated with unit $i$ in period $t$.

$\mathcal{I} \quad$ Index set of generating units.

$\mathcal{I}_{n} \quad$ Index set of the generating units located at bus $n$.

$\mathcal{J} \quad$ Index set of consumers.

$\mathcal{J}_{n} \quad$ Index set of the consumers located at bus $n$.

$\mathcal{L} \quad$ Index set of transmission lines.

$\mathcal{N} \quad$ Index set of buses.

$\mathcal{O}_{i} \quad$ Index set of the generation offer blocks of unit $i$.

$\mathcal{T} \quad$ Index set of time periods.

\section{Functions}

$\mathcal{R}_{i}(\cdot) \quad$ Function representing the right-hand side of the revenue constraint of unit $i$.

\section{Constants}

$C_{b j t}^{d} \quad$ Price of the $b$ th demand block bid by consumer $j$ in period $t$.

$C_{\text {oit }}^{g} \quad$ Price of the oth generation block offered by unit $i$ in period $t$.

$C_{i t}^{n l} \quad$ No-load price offered by unit $i$ in period $t$.

$C_{i t}^{s d} \quad$ Shut-down price offered by unit $i$ in period $t$.

$C_{i t}^{s u} \quad$ Start-up price offered by unit $i$ in period $t$.

$D T_{i} \quad$ Minimum down time of unit $i$.

$f r(l)$ Origin bus of line $l$.

$H \quad$ Sufficiently large positive constant. 
$M_{i}^{f} \quad$ Coefficient of the fixed term of the minimum revenue function offered by unit $i$.

$M_{\text {oit }}^{v} \quad$ Slope of the oth block of the minimum revenue function offered by unit $i$ in period $t$.

$n(i) \quad$ Bus where generating unit $i$ is located.

$n(j) \quad$ Bus where consumer $j$ is located.

$n_{B_{j}} \quad$ Cardinality of $\mathcal{B}_{j}$.

$n_{I} \quad$ Number of generating units.

$n_{i}^{D T} \quad$ Number of periods during which unit $i$ must be initially scheduled off due to its minimum down time constraint.

$n_{i}^{U T} \quad$ Number of periods during which unit $i$ must be initially scheduled on due to its minimum up time constraint.

$n_{i 0}^{o f f}$

Number of periods during which unit $i$ has been scheduled off prior to the first period of the time span (end of period 0 ).

$n_{i 0}^{o n} \quad$ Number of periods during which unit $i$ has been scheduled on prior to the first period of the time span (end of period 0 ).

$n_{J} \quad$ Number of consumers.

$n_{L} \quad$ Number of transmission lines.

$n_{N} \quad$ Number of buses.

$n_{O_{i}} \quad$ Cardinality of $\mathcal{O}_{i}$.

$n_{T} \quad$ Number of periods of the time span.

$\bar{P}_{b j t}^{d} \quad$ Upper bound for $p_{b j t}^{d}$.

$\bar{P}_{j t}^{d} \quad$ Upper bound for $p_{j t}^{d}$.

$\underline{P}_{j t}^{d} \quad$ Lower bound for $p_{j t}^{d}$.

$\bar{P}_{l}^{f} \quad$ Power flow capacity of line $l$.

$P_{i 0}^{g} \quad$ Initial power output of unit $i$.

$\bar{P}_{i t}^{g} \quad$ Upper bound for $p_{i t}^{g}$.

$\underline{P}_{i t}^{g} \quad$ Lower bound for $p_{i t}^{g}$.

$\bar{P}_{\text {oit }}^{g} \quad$ Upper bound for $p_{\text {oit }}^{g}$.

$R_{i}^{d n} \quad$ Ramp-down rate of unit $i$.

$R_{i}^{s d} \quad$ Shut-down ramp rate of unit $i$.

$R_{i}^{s u} \quad$ Start-up ramp rate of unit $i$.

$R_{i}^{u p} \quad$ Ramp-up rate of unit $i$.

to $(l) \quad$ Destination bus of line $l$.

$U T_{i} \quad$ Minimum up time of unit $i$.

$V_{i 0} \quad$ Initial on/off status of unit $i$.

$x_{l} \quad$ Reactance of line $l$.

$\gamma_{i t}^{l o} \quad$ Lower bound for dual variable $\gamma_{i t}^{l o}$.

$\bar{\gamma}_{i t}^{u p} \quad$ Upper bound for dual variable $\gamma_{i t}^{u p}$.

$\bar{\epsilon}_{i t} \quad$ Upper bound for dual variable $\epsilon_{i t}$.

$\bar{\xi}_{i t} \quad$ Upper bound for dual variable $\xi_{i t}$.

$\underline{\sigma}_{i t} \quad$ Lower bound for dual variable $\sigma_{i t}$.

$\underline{\Psi}_{i} \quad$ Lower bound for $\Psi_{i}$.

\section{E. Variables}

$a_{i t} \quad$ Auxiliary variable equal to the product $v_{i t} \gamma_{i t}^{l o}$.

$b_{i t} \quad$ Auxiliary variable equal to the product $v_{i t} \gamma_{i t}^{u p}$.

$c_{i t} \quad$ Auxiliary variable equal to the product $v_{i t} \xi_{i t}$.

$d_{i t} \quad$ Auxiliary variable equal to the product $v_{i t-1} \xi_{i t}$.

$e_{i t} \quad$ Auxiliary variable equal to the product $v_{i t} \epsilon_{i t}$.

$k_{i t} \quad$ Auxiliary variable equal to the product $v_{i t+1} \epsilon_{i t}$.

$p_{b j t}^{d} \quad$ Consumption level awarded to block $b$ bid by consumer $j$ in period $t$.

$p_{j t}^{d} \quad$ Power consumption of consumer $j$ in period $t$.

$p_{l t}^{f} \quad$ Power flow of line $l$ in period $t$.

$p_{i t}^{g} \quad$ Power output of unit $i$ in period $t$.

$p_{o i t}^{g} \quad$ Generation level awarded to block $o$ offered by unit $i$ in period $t$.

$q_{i t} \quad$ Auxiliary variable equal to the product $v_{i t} \sigma_{i t}$.

$r_{i} \quad$ Total revenue earned by unit $i$.

$r_{i}^{\min }$ Minimum revenue that unit $i$ is willing to earn.

$s_{i t}^{s d} \quad$ Shut-down offer cost of unit $i$ in period $t$.

$s_{i t}^{s u} \quad$ Start-up offer cost of unit $i$ in period $t$.

$v_{i t} \quad$ Binary variable that is equal to 1 if unit $i$ is scheduled on in period $t$, being 0 otherwise.

$w_{i t} \quad$ Auxiliary variable equal to the product $v_{i t-1} \sigma_{i t}$.

$z_{i} \quad$ Variable that is equal to 0 if unit $i$ is scheduled off along the time span, being 1 otherwise.

$\delta_{n t} \quad$ Voltage phase angle at bus $n$ in period $t$.

$\Delta r_{i} \quad$ Revenue margin of unit $i$.

$\lambda_{t}^{a v} \quad$ Load-weighted average locational marginal price for energy in period $t$.

$\Psi_{i} \quad$ Declared profit of unit $i$.

\section{F. Dual Variables}

$\alpha_{i t} \quad$ Dual variable associated with the definition of $p_{i t}^{g}$ as the sum of the generation levels awarded to the offer blocks.

$\beta_{\text {oit }}^{l o} \quad$ Dual variable associated with the constraint imposing the lower bound for $p_{\text {oit }}^{g}$.

$\beta_{\text {oit }}^{u p} \quad$ Dual variable associated with the constraint imposing the upper bound for $p_{\text {oit }}^{g}$.

$\gamma_{i t}^{l o} \quad$ Dual variable associated with the constraint imposing the lower bound for $p_{i t}^{g}$.

$\gamma_{i t}^{u p} \quad$ Dual variable associated with the constraint imposing the upper bound for $p_{i t}^{g}$.

$\epsilon_{i t}$ Dual variable associated with the shut-down ramp rate constraint of unit $i$ in period $t$.

$\theta_{j t} \quad$ Dual variable associated with the definition of $p_{j t}^{d}$ as the sum of the consumption levels awarded to the demand bid blocks.

$\lambda_{n t} \quad$ Locational market-clearing price for energy at bus $n$ in period $t$.

$\mu_{b j t}^{l o} \quad$ Dual variable associated with the constraint imposing the lower bound for $p_{b j t}^{d}$. 

$\mu_{b j t}^{u p}$
Dual variable associated with the constraint impos- ing the upper bound for $p_{b j t}^{d}$.
$\nu_{l t} \quad$ Dual variable associated with the definition of $p_{l t}^{f}$ in terms of $\delta_{n t}$.
$\xi_{i t} \quad$ Dual variable associated with the constraint mod- eling the ramp-up and start-up ramp rates of unit $i$ in period $t$.
$\rho_{j t}^{l o} \quad$ Dual variable associated with the constraint impos- ing the lower bound for $p_{j t}^{d}$.
$\rho_{j t}^{u p} \quad$ Dual variable associated with the constraint impos- ing the upper bound for $p_{j t}^{d}$.
$\sigma_{i t} \quad$ Dual variable associated with the ramp-down rate constraint of unit $i$ in period $t$.
$\phi_{l t}^{l o} \quad$ Dual variable associated with the constraint impos- ing the lower bound for $p_{l t}^{f}$.
$\phi_{l t}^{u p} \quad$ Dual variable associated with the constraint impos- ing the upper bound for $p_{l t}^{f}$.

\section{INTRODUCTION}

$\mathbf{R}$ EVENUE sufficiency is a desirable property in electricity markets by which revenues earned from awarded offers should cover the offer costs [2], [3]. Unfortunately, currently implemented auction designs relying on both unit commitment models and marginal-pricing-based remuneration [4], [5] do not guarantee the fulfillment of revenue sufficiency [2], [6]. Under such market designs, revenues from selling energy are determined by the products of the generation levels and marginal market-clearing prices for energy resulting from an economic dispatch problem with scheduling variables fixed at their optimal values. Due to the presence of nonconvexities associated with power system operation, such as no-load, startup, and shut-down offers, as well as indivisibilities in power generation and consumption, offer costs may exceed revenues. This issue may be stressed by the growing production levels from intermittent renewable-based generating units, which are characterized by essentially zero marginal costs but require the scheduling of conventional plants to cope with unexpected network congestions and ramping events.

The revenue insufficiency of nonconvex electricity markets under marginal pricing can be addressed in two different ways:

1) By adopting an alternative remuneration scheme leading to a new market settlement [7]-[19] either by 1) uplifting the payments resulting from locational marginal pricing, 2) using other types of uplift, 3) relying on a pricing scheme different from marginal pricing, or 4) combining some of them. The strategy adopted in US electricity markets belongs to this class and consists in the implementation of out-of-market financial mechanisms as lump-sum monetary transfers between market agents [7], [8], [11], also known as offer cost compensations or make-whole payments, under locational marginal pricing. More specifically, producers that, as a result of the market-clearing process, incur economic losses, i.e., negative declared profits, are paid ex post the difference between the offer costs and the revenues earned. Additionally, in the presence of opportunity costs, uplift payments incentivize the attainment of an equilibrium solution.

2) Through a tighter formulation of the market-clearing procedure explicitly setting bounds on either the revenues or the offer-based profits earned by producers [16], [17], [20]-[31], thereby resulting in a revenueconstrained auction model. The strategy adopted in national electricity markets across Europe falls within this category and consists in considering a marginal pricing scheme and extending the constraint set of the auction model to include terms related to revenues from selling energy that are referred to as minimum income conditions. Relevant examples are the Iberian electricity market [20] and APX [21]. Moreover, such revenue constraints are also explicitly accounted for in a somewhat related day-ahead auction model dealing with cross-border trading at a pan-European level [32]. Such an auction model is a previous step towards the creation of a single electricity market replacing the national ones, as a consequence of the increasing commitment to a common European energy policy [33]. It should be noted that the resulting pan-European market will be greater than those of Midwest ISO and PJM.

An important policy issue that is beyond the scope of this paper is the discussion on which alternative is the best option. Whether guaranteeing revenue sufficiency through alternative remuneration schemes such as the make-whole payments currently implemented in US markets, or through the extension of the constraint set of the auction models with revenue constraints, as done in Europe, has raised a longstanding debate in industry and academia due to the pros and cons of both approaches. Revenue-unconstrained models with make-whole payments are far simpler from a mathematical perspective and yield the maximum attainable level of social welfare. In contrast, the required compensations may give rise to price discrimination among market participants, may provide inadequate economic signals, and require the identification of the agents paying for such uplifts, which may be controversial [11], [15], [16]. On the other hand, revenueconstrained models are not discriminatory and maintain the desirable economic properties associated with marginal pricing. In addition, offers including too high bounds on revenues or profits increase the possibility of not being scheduled. Therefore, some researchers argue that honest offering by suppliers is promoted [34] so that generation offers reflect actual costs. Unfortunately, the attainment of such benefits requires sacrificing social welfare and transparency, solving a mathematically challenging optimization problem for which no exact solution methodology is currently available, and facing the potential exercise of market power by producers. The implementation of instances of both alternatives in current industry practice reveals the lack of consensus on this debate.

Motivated by the undeniable practical interest and the modeling and methodological challenges posed, this paper is focused on an instance of the alternative adopted in Europe, namely revenue- and network-constrained market clearing for energy in a day-ahead pool-based electricity market under marginal pricing. Market-clearing procedures for energy are 
implemented by system operators to determine the set of awarded generation offers and demand bids for energy maximizing the level of declared social welfare. In addition, locational market-clearing prices for energy also result from the optimization process. Here, market clearing is implemented under the standard marginal pricing scheme [35] while considering demand-side bidding, the effect of the transmission network, as well as generation-related features such as production limits, ramp rates, minimum up and down times, and revenue constraints. The modeling framework is general to incorporate the aforementioned two instances of revenue constraints, thereby being suitable for day-ahead electricity markets currently implemented worldwide. Similar to previous related works [16], [17], [22]-[31], the analysis of market power abuse is beyond the scope of this two-paper series.

Unlike revenue-unconstrained auction designs, such as those implemented in US electricity markets, which are typically formulated as instances of mixed-integer linear programming [4], [36], the revenue-constrained models routinely solved in Europe [20], [21] belong to the class of price-based market clearing [37] where market-clearing prices become decision variables of the optimization. This aspect drastically complicates the solution of the resulting problem in two respects: 1) market-clearing prices may themselves result from an optimization process, as is the case under marginal pricing; and 2) revenue constraints include bilinear and hence highly nonconvex terms involving products of two continuous decision variables, namely power outputs and market-clearing prices, thereby leading to mixed-integer nonlinear optimization models for which exact solutions are in general unavailable.

Such challenges have triggered extensive research on this topic [16], [17], [20]-[31]. However, most works may be of reduced practical applicability given the use of an optimization goal different from the widely accepted declared social welfare maximization [22]-[25], [28], [31]. Therefore, within the context of current industry practice where the optimization process is driven by declared social welfare maximization, relevant works on revenue-constrained auction models are [16], [17], [26], [27], [29], [30].

In [26], [27], Motto and Galiana formulated a singleperiod and single-bus version of revenue-constrained market clearing as a mathematical program with equilibrium constraints [38]. A mixed-integer linear equivalent was derived by using Karush-Kuhn-Tucker (KKT) optimality conditions and a linearization scheme requiring additional binary variables. Moreover, bilinear revenue terms were equivalently linearized by introducing the concepts of augmented cost and augmented revenue. Unfortunately, the solution approach is not suitable for auction models incorporating transmission network constraints or inter-temporal operational constraints.

In [29], [30], García-Bertrand et al. dealt with a competitive market near-equilibrium including minimum profit conditions. The proposed solution approach consisted in appending the revenue-related constraints to the set of optimality conditions associated with the corresponding revenue-unconstrained auction. Subsequently, bilinear revenue terms were addressed by either 1) an iterative procedure based on a successive over-relaxation [29] or 2) as done in [30], a computationally expensive approximation based on Schur's decomposition [39] requiring additional binary variables. As recognized by the authors, both approaches may yield suboptimal or even infeasible solutions.

In [16], Ruiz et al. proposed a revenue-constrained auction in a single-period setting wherein the presence of nonconvexities was addressed by using a procedure based on a primal-dual transformation. This approach features two shortcomings. First, this methodology may lead to solutions different from those yielded by an economic dispatch problem and, as a consequence, the resulting locational market-clearing prices may be inconsistent with the theory of marginal pricing. Moreover, the binary expansion approach [40] used to linearize revenue terms is a computationally expensive approximation requiring extra binary variables.

In [17], discretely constrained, mixed linear complementarity problems were solved by relaxing both integrality and complementarity to build feasible problems. Within this framework, Gabriel et al. addressed a revenue- and networkconstrained day-ahead auction model including price uplifts and a binary-expansion-based approach for bilinear revenue terms. Similar to [16], the methodology relies on an inexact and computationally inefficient approximation and the desirable consistency with the widely adopted economic-dispatchbased marginal pricing is not guaranteed.

Regarding industry practice, several real-life electricity markets deal with revenue constraints in day-ahead market clearing [20], [21]. A relevant approach for this instance of price-based auction can be found in the operational rules of the Iberian day-ahead electricity market, where an ad-hoc heuristic is described [20]. However, this approach does not guarantee optimality and may even terminate without attaining feasibility, as shown in the companion paper [1] and in [27].

The thrust of this paper is the proposal of an original and exact approach based on bilevel programming [41] to effectively address the real-life instances of revenue-constrained day-ahead market clearing under marginal pricing that have been implemented in several European electricity markets [20], [21] for over a decade and that are currently solved without even ensuring the attainment of feasibility [20]. Moreover, the proposed approach improves upon previously reported solutions for revenue-constrained market clearing [16], [17], [26], [27], [29], [30], which rely on simplified models or either inexact or computationally expensive methodologies.

The benefits of using bilevel programming are threefold. First, locational marginal prices, i.e., sensitivities, can be properly characterized without requiring any modeling simplifications. Furthermore, this sound mathematical-programmingbased approach relies on well-grounded properties related to optimality and feasibility. In addition, the particular conditions featured by the proposed bilevel program allow its transformation to a single-level equivalent suitable for commercially available software.

Based on the findings of [37], the schedule of generating units is determined in the upper-level problem, whereas the lower-level problem is associated with dispatching decisions including levels of awarded offers and bids, network-related variables, as well as the corresponding locational marginal 
prices. In addition, primal and dual information from the lower level is used to compute producer revenues from selling energy. The proposed bilevel programming framework gives rise to relevant distinctive modeling aspects over previous works [16], [17], [26], [27], [30]. First, this paper differs from [26], [27] due to the explicit consideration of the effect of the transmission network. Unlike [16], [17], the economically desirable uplift-free remuneration scheme relying on economic-dispatch-based marginal pricing [35] is preserved. Moreover, in contrast to [16], [17], [26], [27], [30], intertemporal operational constraints such as minimum up and down times as well as ramping rates are explicitly accounted for. As another salient modeling feature, the proposed bilevel programming framework allows accommodating any practical instance of generation revenue constraints such as minimum revenue conditions and minimum profit constraints.

The resulting problem is formulated as a mixed-integer nonlinear bilevel program with bilinear revenue terms for which no exact solution technique is currently available. The key idea of the proposed solution approach is to convert the original problem into an equivalent single-level mixed-integer linear program. To that end, a two-step procedure is proposed. First, based on practical modeling aspects that render the lowerlevel problem an instance of linear programming, an effective primal-dual transformation [42] is applied to recast the original bilevel program as a single-level equivalent with bilinear terms. Subsequently, and as a major distinctive methodological feature over [16], [17], [26], [27], [29], [30], bilinear terms are handled by a novel, exact, and computationally inexpensive linearization scheme without requiring the addition of extra binary variables. This linearization is based on duality theory of linear programming, KKT optimality conditions, and integer algebra results. The resulting single-level equivalent is an instance of mixed-integer linear programming. Hence, finite convergence to optimality is guaranteed while providing a measure of the distance to optimality along the optimization process [43]. Moreover, off-the-shelf software based on the standard branch-and-cut algorithm is readily available [44], which is beneficial for practical implementation purposes.

Thus, this paper features advantageous modeling and methodological novelties with respect to the heuristic used in industry practice [20] and previous relevant works [16], [17], [26], [27], [29], [30]. The modeling novelties are 1) the joint consideration of marginal pricing, network constraints, demand-side bidding, and a multiperiod setting precisely incorporating inter-temporal operational constraints; and 2) the flexibility to characterize both minimum revenue conditions and minimum profit constraints. The methodological novelty is the transformation of the original mixed-integer nonlinear bilevel problem to a computationally effective mixedinteger linear equivalent. This conversion, aside from using a sound primal-dual transformation, relies on a new linearization scheme for bilinear revenue terms involving the products of two continuous decision variables that, unlike available linearization schemes [39], [40], is exact and does not require additional binary variables.

The main contributions of this paper are twofold:
1) From a modeling perspective, a novel formulation is presented to account for generation revenue constraints in pool-based day-ahead market-clearing procedures under marginal pricing, such as those currently implemented across Europe. The resulting model, which includes the effect of the transmission network, demand-side bidding, and inter-temporal operational constraints, is formulated as a mixed-integer nonlinear bilevel program with bilinear terms. It is worth emphasizing that explicitly formulating time-varying locational marginal prices as decision variables of the optimization problem is of utmost importance because it permits the development of mathematical-programming-based solutions to this complex problem, such as the one reported here.

2) From a methodological perspective, this paper provides, for the first time in the technical literature, an effective solution approach based on a primal-dual transformation and the exact conversion of bilinear revenue terms into equivalent linear expressions, without requiring extra binary variables. As a result, the original mixed-integer nonlinear bilevel program with bilinear terms is recast as a single-level mixed-integer linear equivalent that overcomes the shortcomings of available solutions in industry practice and in the technical literature.

It should be noted that the tool presented here, aside from addressing the unresolved challenges posed in practice by revenue-constrained market clearing, provides valuable information for the debate on the best way to ensure revenue sufficiency in nonconvex electricity markets under marginal pricing. As an example, the proposed approach may help system operators and regulators to accurately monitor market participants' behavior and to precisely examine the long-term implications of this trading scheme. Such analyses may require dynamic game theoretical models or equilibrium-based models wherein the proposed tool would play a key role.

The remainder of this paper is organized as follows. Section II presents the problem formulation. Section III describes the solution approach. Relevant conclusions are provided in Section IV. Finally, for the sake of completeness, wellknown linear expressions for minimum up and down times, shutdowns, and start-ups are formulated in the Appendix. Furthermore, results from several case studies are presented and discussed in the companion paper [1]. It should be noted that the adoption of a two-part setting is intended to clearly convey the methodological contributions on a step-by-step basis. This layout also allows comprehensively illustrating the numerical behavior of the proposed approach and its superiority over existing methodologies from the perspectives of both solution quality and computational burden.

\section{PRoblem Formulation}

The incorporation of revenue constraints in day-ahead market clearing requires the selection of a pricing scheme to characterize market-clearing prices. Based on worldwide industry practice [4], [5] and relevant references on revenueconstrained market clearing [26], [27], [29], [30], a marginal pricing scheme relying on a single nodal price for energy in 
each period [35] is adopted. Thus, locational market-clearing prices are hereinafter referred to as locational marginal prices.

It is worth emphasizing that the theory of marginal pricing relies on the assumption of convexity of the optimization. Unfortunately, convexity is not held in market clearing due to the presence of binary variables, which are required to model minimum power outputs different from zero, minimum up and down times, shutdowns, and start-ups. This issue has triggered extensive research on nonconvex pricing for generation scheduling problems [12], [14], [16]. A widely adopted solution, however, is to derive marginal prices from a multiperiod economic dispatch or optimal power flow driven by declared social welfare maximization wherein binary scheduling variables have been fixed at their optimal values [4], [5]. Thus, as prescribed in [35], locational energy prices for a given feasible schedule are characterized in terms of the Lagrange multipliers or dual variables associated with the nodal power balance equations. In other words, the definition of the vector of market-clearing prices under marginal pricing is an optimization problem itself. This optimization also provides the optimal generation levels associated with the optimal generation schedule. Therefore, since locational marginal prices and generation levels are both dependent on the set of binary scheduling variables, the above-described practical procedure to obtain marginal prices in nonconvex auctions is also suitable for the nonconvexity of bilinear generation revenue terms involving products of generation levels and prices.

Hence, based on [37], the proposed revenue- and networkconstrained day-ahead auction design can be formulated as the following mixed-integer nonlinear bilevel programming problem:

$$
\begin{aligned}
\underset{\substack{r_{i}, s_{i t}^{s d}, s_{i t}^{s u}, v_{i t}, z_{i}}}{\operatorname{Maximize}} \sum_{j \in \mathcal{J}} \sum_{b \in \mathcal{B}_{j}} \sum_{t \in \mathcal{T}} C_{b j t}^{d} p_{b j t}^{d}- \\
\sum_{i \in \mathcal{I}} \sum_{t \in \mathcal{T}}\left(\sum_{o \in \mathcal{O}_{i}} C_{o i t}^{g} p_{\text {oit }}^{g}+s_{i t}^{s d}+s_{i t}^{s u}+C_{i t}^{n l} v_{i t}\right)
\end{aligned}
$$

subject to:

$$
\begin{aligned}
& s_{i t}^{s d}, s_{i t}^{s u}, v_{i t} \in \mathcal{F}_{i t} ; \forall i \in \mathcal{I}, \forall t \in \mathcal{T} \\
& v_{i t} \in\{0,1\} ; \forall i \in \mathcal{I}, \forall t \in \mathcal{T} \\
& 0 \leq z_{i} \leq 1 ; \forall i \in \mathcal{I} \\
& z_{i} \geq v_{i t} ; \forall i \in \mathcal{I}, \forall t \in \mathcal{T} \\
& z_{i} \leq \sum_{t \in \mathcal{T}} v_{i t} ; \forall i \in \mathcal{I} \\
& r_{i}=\sum_{t \in \mathcal{T}} p_{i t}^{g} \lambda_{n(i) t} ; \forall i \in \mathcal{I} \\
& r_{i} \geq \mathcal{R}_{i}\left(p_{\text {oit }}^{g}, s_{i t}^{s d}, s_{i t}^{s u}, v_{i t}, z_{i}\right) ; \forall i \in \mathcal{I}
\end{aligned}
$$

where optimal consumption levels $p_{b j t}^{d}$, generation levels $p_{i t}^{g}$ and $p_{\text {oit }}^{g}$, and dual variables $\lambda_{n t}$ are obtained from the following multiperiod dc optimal power flow:

$$
\underset{\substack{p_{b j t}^{d}, p_{j t}^{d}, p_{l t}^{f}, p_{i t}^{g}, p_{\text {oit }}^{g}, \delta_{n t}}}{\operatorname{Maximize}} \sum_{j \in \mathcal{J}} \sum_{b \in \mathcal{B}_{j}} \sum_{t \in \mathcal{T}} C_{b j t}^{d} p_{b j t}^{d}-\sum_{i \in \mathcal{I}} \sum_{o \in \mathcal{O}_{i}} \sum_{t \in \mathcal{T}} C_{o i t}^{g} p_{o i t}^{g}
$$

subject to:

$$
\begin{aligned}
& \sum_{j \in \mathcal{J}_{n}} p_{j t}^{d}=\sum_{i \in \mathcal{I}_{n}} p_{i t}^{g}+\sum_{l \mid t o(l)=n} p_{l t}^{f} \\
& \quad-\sum_{l \mid f r(l)=n} p_{l t}^{f}:\left(\lambda_{n t}\right) ; \forall n \in \mathcal{N}, \forall t \in \mathcal{T} \\
& p_{l t}^{f}=\frac{1}{x_{l}}\left(\delta_{f r(l) t}-\delta_{t o}(l) t\right):\left(\nu_{l t}\right) ; \forall l \in \mathcal{L}, \forall t \in \mathcal{T} \\
& -\bar{P}_{l}^{f} \leq p_{l t}^{f} \leq \bar{P}_{l}^{f}:\left(\phi_{l t}^{l o}, \phi_{l t}^{u p}\right) ; \forall l \in \mathcal{L}, \forall t \in \mathcal{T} \\
& \underline{P}_{i t}^{g} v_{i t} \leq p_{i t}^{g} \leq \bar{P}_{i t}^{g} v_{i t}:\left(\gamma_{i t}^{l o}, \gamma_{i t}^{u p}\right) ; \forall i \in \mathcal{I}, \forall t \in \mathcal{T} \\
& \underline{P}_{j t}^{d} \leq p_{j t}^{d} \leq \bar{P}_{j t}^{d}:\left(\rho_{j t}^{l o}, \rho_{j t}^{u p}\right) ; \forall j \in \mathcal{J}, \forall t \in \mathcal{T} \\
& p_{i t}^{g}=\sum_{o \in \mathcal{O}_{i}} p_{o i t}^{g}:\left(\alpha_{i t}\right) ; \forall i \in \mathcal{I}, \forall t \in \mathcal{T} \\
& 0 \leq p_{o i t}^{g} \leq \bar{P}_{o i t}^{g}:\left(\beta_{o i t}^{l o}, \beta_{o i t}^{u p}\right) ; \forall i \in \mathcal{I}, \forall o \in \mathcal{O}_{i}, \forall t \in \mathcal{T} \\
& p_{j t}^{d}=\sum_{b \in \mathcal{B}_{j}} p_{b j t}^{d}:\left(\theta_{j t}\right) ; \forall j \in \mathcal{J}, \forall t \in \mathcal{T} \\
& 0 \leq p_{b j t}^{d} \leq \bar{P}_{b j t}^{d}:\left(\mu_{b j t}^{l o}, \mu_{b j t}^{u p}\right) ; \forall j \in \mathcal{J}, \forall b \in \mathcal{B}_{j}, \forall t \in \mathcal{T} \\
& p_{i t}^{g} \leq p_{i t-1}^{g}+R_{i}^{u p} v_{i t-1}+R_{i}^{s u}\left(v_{i t}-v_{i t-1}\right) \\
& \quad+\bar{P}_{i t}^{g}\left(1-v_{i t}\right):\left(\xi_{i t}\right) ; \forall i \in \mathcal{I}, \forall t \in \mathcal{T} \\
& p_{i t}^{g} \geq p_{i t-1}^{g}-R_{i}^{d n} v_{i t}-R_{i}^{s d}\left(v_{i t-1}-v_{i t}\right) \\
& \quad-\bar{P}_{i t}^{g}\left(1-v_{i t-1}\right):\left(\sigma_{i t}\right) ; \forall i \in \mathcal{I}, \forall t \in \mathcal{T} \\
& p_{i t}^{g} \leq R_{i}^{s d}\left(v_{i t}-v_{i t+1}\right)+\bar{P}_{i t}^{g} v_{i t+1}:\left(\epsilon_{i t}\right) ; \\
& \quad \forall i=1 \ldots n_{T}-1 .
\end{aligned}
$$

For the sake of consistency, hourly time periods are considered; $\sum_{b \in \mathcal{B}_{j}} \bar{P}_{b j t}^{d}=\bar{P}_{j t}^{d}, \forall j \in \mathcal{J}, \forall t \in \mathcal{T}$; and $\sum_{o \in \mathcal{O}_{i}} \bar{P}_{\text {oit }}^{g}=$ $\bar{P}_{i t}^{g}, \forall i \in \mathcal{I}, \forall t \in \mathcal{T}$.

The bilevel model (1)-(21) comprises two optimization levels, namely the upper level (1)-(8) and the lower level (9)-(21). Upper-level decision variables are producer revenues $r_{i}$, shut-down offers $s_{i t}^{s d}$, start-up offers $s_{i t}^{s u}$, on/off statuses of generating units $v_{i t}$, and variables $z_{i}$ associated with revenue constraints. Lower-level decision variables are power flows $p_{l t}^{f}$, voltage phase angles $\delta_{n t}$, power outputs $p_{i t}^{g}$, power consumptions $p_{j t}^{d}$, and awarded levels of generation offer and demand bid blocks $p_{\text {oit }}^{g}$ and $p_{b j t}^{d}$, respectively, whereas dual variables $\lambda_{n t}$ are an outcome of the lower-level problem.

The goal of the upper-level problem is to maximize the revenue-constrained declared social welfare (1) evaluated at the optimal values of consumption levels $p_{b j t}^{d}$, generation levels $p_{i t}^{g}$ and $p_{\text {oit }}^{g}$, and dual variables $\lambda_{n t}$. The objective function maximized in (1) comprises five terms respectively related to 1) demand bids, 2) generation energy offers, 3) generation shut-down offers, 4) generation start-up offers, and 5) generation no-load offers. As is customary in industry [20], [21], stepwise linear bids and offers for energy are considered.

The feasibility set $\mathcal{F}_{i t}$ in (2) includes minimum up and down times, shutdowns, and start-ups, which are modeled using mixed-integer linear programming [45], as formulated 
in the Appendix. Expressions (3) impose the integrality of scheduling variables $v_{i t}$.

Expressions (4)-(6) characterize variables $z_{i}$, which identify those generators whose revenue constraints must be enforced, i.e., those with awarded offers along the time span. Thus, $z_{i}$ is equal to 1 if unit $i$ is scheduled on in at least one period and, hence, the corresponding revenue constraint is imposed. On the other hand, $z_{i}$ is equal to 0 for those units that are scheduled off along the scheduling horizon, for which the corresponding revenue constraints are disregarded. Therefore, $z_{i}$ is a binaryvalued variable equal to the maximum of $v_{i t}$ over all periods. Note, however, that $z_{i}$ can be equivalently formulated as a continuous variable lying in the interval $[0,1]$, as modeled in (4), that is greater than or equal to every $v_{i t}$ along the time span (5), and that is less than or equal to the summation of $v_{i t}$ over all time periods (6). If unit $i$ is scheduled off in all periods, i.e., $v_{i t}=0, \forall t \in \mathcal{T}, z_{i}=\max \{0, \ldots, 0\}=$ 0 . For this case, (4) and (6) would yield the same result, as desired. In contrast, if some $v_{i t}$ is equal to 1 for unit $i, z_{i}=$ $\max _{t \in \mathcal{T}}\left\{v_{i t}\right\}=1$, which results from (4) and (5).

The bilinear revenue terms from selling energy are defined in (7), where both primal and dual variables of the lower-level problem are involved. Expressions (8) represent generation revenue constraints. Note that $\mathcal{R}_{i}(\cdot)$ may adopt different forms to accommodate any instance of revenue-constrained auction designs such as those incorporating minimum revenue conditions or minimum profit constraints, as described next:

1) Minimum revenue conditions impose that the total revenue earned by each scheduled unit must be greater than or equal to a level determined by the corresponding minimum revenue function submitted as part of its generation offer. Thus, the minimum revenue function of unit $i$, which corresponds to $\mathcal{R}_{i}(\cdot)$ in (8), yields the minimum revenue this unit is willing to earn for each awarded energy offer. Based on current industry practice [20], minimum revenue conditions can be incorporated in (8) as follows:

$$
r_{i} \geq M_{i}^{f} z_{i}+\sum_{o \in \mathcal{O}_{i}} \sum_{t \in \mathcal{T}} M_{\text {oit }}^{v} p_{\text {oit }}^{g} ; \forall i \in \mathcal{I} .
$$

For each unit $i$, the right-hand side of (22) is the minimum revenue function, which comprises two terms: 1) a fixed term characterized by parameter $M_{i}^{f}$, and 2) an energy-dependent variable term where parameters $M_{\text {oit }}^{v}$ are the slopes related to the energy offer blocks. Note that if $z_{i}=0$, and hence $p_{\text {oit }}^{g}=0, \forall o \in \mathcal{O}_{i}$, $\forall t \in \mathcal{T}$, the minimum revenue is equal to 0 , i.e., the revenue condition does not constrain the optimization.

2) Minimum profit constraints impose that the declared profit of each scheduled unit $i, \Psi_{i}$, must be greater than or equal to a lower bound $\underline{\Psi}_{i}$. The declared profit of unit $i$ is defined as the revenue earned by this unit minus its generation offer cost:

$$
\begin{aligned}
\Psi_{i}= & r_{i}-\sum_{o \in \mathcal{O}_{i}} \sum_{t \in \mathcal{T}} C_{o i t}^{g} p_{o i t}^{g} \\
& -\sum_{t \in \mathcal{T}}\left(s_{i t}^{s d}+s_{i t}^{s u}+C_{i t}^{n l} v_{i t}\right) ; \forall i \in \mathcal{I}
\end{aligned}
$$

Thus, minimum profit constraints are modeled by replacing (8) as follows:

$$
\begin{aligned}
& r_{i}-\sum_{o \in \mathcal{O}_{i}} \sum_{t \in \mathcal{T}} C_{o i t}^{g} p_{o i t}^{g}-\sum_{t \in \mathcal{T}}\left(s_{i t}^{s d}\right. \\
& \left.\quad+s_{i t}^{s u}+C_{i t}^{n l} v_{i t}\right) \geq \underline{\Psi}_{i} z_{i}-H\left(1-z_{i}\right) ; \forall i \in \mathcal{I}
\end{aligned}
$$

where $H$ is a sufficiently large positive constant used to discard minimum profit constraints for those units that are scheduled off throughout the time span.

It is worth pointing out that both (22) and (24) are mixedinteger linear expressions.

The lower-level problem is a multiperiod optimal power flow particularized for the optimal values of the scheduling variables $v_{i t}$. This optimization problem is also driven by the maximization of the revenue-constrained declared social welfare (9). Note that the shut-down, start-up, and no-load terms included in (1) are dropped in the objective function (9) because those terms become constant for given values of $v_{i t}$. Expressions (10) are the nodal power balance equations, where each associated Lagrange multiplier or dual variable $\lambda_{n t}$ represents the sensitivity of the optimal revenue-constrained declared social welfare with respect to a small perturbation in the corresponding power balance constraint for the values of scheduling variables $v_{i t}$ determined in the upper level. Thus, according to [35], $\lambda_{n t}$ is the marginal price for energy at bus $n$ in period $t$. Using a dc load flow model, constraints (11) characterize line power flows in terms of voltage phase angles. Constraints (12) impose the transmission capacity limits across the transmission network. Production and consumption limits are respectively set in (13) and (14). The block structures of generation offers and demand bids are respectively characterized through constraints (15)-(16) and (17)-(18). Finally, ramping rates are modeled by (19)-(21) as described in [45].

Problem (1)-(21) is a mixed-integer bilevel program with two particular features, namely 1) bilinear terms involving products of two continuous decision variables are included in (7), and 2) the lower-level problem (9)-(21) is parameterized in terms of the upper-level binary variables $v_{i t}$ in such a way that this optimization is a linear program.

\section{SOlution APPROACH}

The proposed solution approach consists in reformulating the mixed-integer nonlinear bilevel program with bilinear terms (1)-(21) as a single-level mixed-integer linear equivalent according to the following two-step procedure.

\section{A. Step 1: Single-Level Equivalent with Bilinear Terms}

Based on the linearity of the lower level (9)-(21), the original bilevel program can be converted into a mixed-integer nonlinear equivalent by using results from duality theory of linear programming [46]. As shown in [42], the lowerlevel problem can be equivalently replaced with its primal feasibility constraints (10)-(21), its dual feasibility constraints, and the equality corresponding to the strong duality theorem in which the lower-level primal and dual objective functions are equated. This primal-dual transformation yields the following single-level mixed-integer program including bilinear terms: 


$$
\begin{aligned}
& \underset{p_{b j t}^{d}, p_{j t}^{d}, p_{l t}^{f}, p_{i t}^{g},}{\operatorname{Maximize}} \quad \sum_{j \in \mathcal{J}} \sum_{b \in \mathcal{B}_{j}} \sum_{t \in \mathcal{T}} C_{b j t}^{d} p_{b j t}^{d} \\
& \begin{array}{l}
p_{\text {oit }}^{g}, r_{i}, s_{i t}^{s d}, s_{i t}^{s u}, \\
v_{i t}, z_{i}, \alpha_{i t}, \beta_{o i t}^{l o}, \\
\beta_{o i t}^{u p}, \gamma_{i t}^{l o}, \gamma_{i t}^{u p}, \delta_{n t},
\end{array}, \quad-\sum_{i \in \mathcal{I}} \sum_{t \in \mathcal{T}}\left(\sum_{o \in \mathcal{O}_{i}} C_{o i t}^{g} p_{o i t}^{g}+s_{i t}^{s d}+s_{i t}^{s u}\right. \\
& \epsilon_{i t}, \theta_{j t}, \lambda_{n t}, \mu_{b j t}^{l o} \text {, } \\
& \mu_{b j t}^{u p}, \nu_{l t}, \xi_{i t}, \rho_{j t}^{l o} \text {, } \\
& \rho_{j t}^{u p}, \sigma_{i t}, \phi_{l t}^{l o}, \phi_{l t}^{u p} \\
& \left.+C_{i t}^{n l} v_{i t}\right)
\end{aligned}
$$

subject to:

Constraints (2)-(6) and (8)

$r_{i}=\sum_{t \in \mathcal{T}} p_{i t}^{g} \lambda_{n(i) t} ; \forall i \in \mathcal{I}$

Constraints (10)-(21)

$-\lambda_{n(i) t}+\gamma_{i t}^{l o}+\gamma_{i t}^{u p}+\xi_{i t}-\xi_{i t+1}+\sigma_{i t}-\sigma_{i t+1}$

$+\epsilon_{i t}+\alpha_{i t}=0 ; \forall i \in \mathcal{I}, \forall t=1 \ldots n_{T}-1$

$-\lambda_{n(i) n_{T}}+\gamma_{i n_{T}}^{l o}+\gamma_{i n_{T}}^{u p}+\xi_{i n_{T}}+\sigma_{i n_{T}}$

$+\alpha_{i n_{T}}=0 ; \forall i \in \mathcal{I}$

$-\alpha_{i t}+\beta_{\text {oit }}^{\text {lo }}+\beta_{\text {oit }}^{u p}=-C_{\text {oit }}^{g} ; \forall i \in \mathcal{I}, \forall o \in \mathcal{O}_{i}, \forall t \in \mathcal{T}$ (31)

$\lambda_{n(j) t}+\rho_{j t}^{l o}+\rho_{j t}^{u p}+\theta_{j t}=0 ; \forall j \in \mathcal{J}, \forall t \in \mathcal{T}$

$-\theta_{j t}+\mu_{b j t}^{l o}+\mu_{b j t}^{u p}=C_{b j t}^{d} ; \forall j \in \mathcal{J}, \forall b \in \mathcal{B}_{j}, \forall t \in \mathcal{T}$

$\nu_{l t}+\phi_{l t}^{l o}+\phi_{l t}^{u p}+\lambda_{f r(l) t}-\lambda_{t o(l) t}=0 ; \forall l \in \mathcal{L}, \forall t \in \mathcal{T}$ (34)

$-\sum_{l \mid f r(l)=n} \frac{1}{x_{l}} \nu_{l t}+\sum_{l \mid t o(l)=n} \frac{1}{x_{l}} \nu_{l t}=0 ; \forall n \in \mathcal{N}, \forall t \in \mathcal{T}$

$\gamma_{i t}^{l o}, \sigma_{i t} \leq 0, \gamma_{i t}^{u p}, \xi_{i t} \geq 0 ; \forall i \in \mathcal{I}, \forall t \in \mathcal{T}$

$\epsilon_{i t} \geq 0 ; \forall i \in \mathcal{I}, \forall t=1 \ldots n_{T}-1$

$\rho_{j t}^{l o} \leq 0, \rho_{j t}^{u p} \geq 0 ; \forall j \in \mathcal{J}, \forall t \in \mathcal{T}$

$\phi_{l t}^{l o} \leq 0, \phi_{l t}^{u p} \geq 0 ; \forall l \in \mathcal{L}, \forall t \in \mathcal{T}$

$\beta_{\text {oit }}^{\text {lo }} \leq 0, \beta_{\text {oit }}^{\text {up }} \geq 0 ; \forall i \in \mathcal{I}, \forall o \in \mathcal{O}_{i}, \forall t \in \mathcal{T}$

$\mu_{b j t}^{l o} \leq 0, \mu_{b j t}^{u p} \geq 0 ; \forall j \in \mathcal{J}, \forall b \in \mathcal{B}_{j}, \forall t \in \mathcal{T}$

$\sum_{j \in \mathcal{J}} \sum_{b \in \mathcal{B}_{j}} \sum_{t \in \mathcal{T}} C_{b j t}^{d} p_{b j t}^{d}-\sum_{i \in \mathcal{I}} \sum_{o \in \mathcal{O}_{i}} \sum_{t \in \mathcal{T}} C_{o i t}^{g} p_{\text {oit }}^{g}=$

$\sum_{l \in \mathcal{L}} \sum_{t \in \mathcal{T}} \bar{P}_{l}^{f}\left(\phi_{l t}^{u p}-\phi_{l t}^{l o}\right)$

$+\sum_{i \in \mathcal{I}} \sum_{t \in \mathcal{T}}\left(\underline{P}_{i t}^{g} v_{i t} \gamma_{i t}^{l o}+\bar{P}_{i t}^{g} v_{i t} \gamma_{i t}^{u p}+\sum_{o \in \mathcal{O}_{i}} \bar{P}_{o i t}^{g} \beta_{o i t}^{u p}\right)$

$+\sum_{j \in \mathcal{J}} \sum_{t \in \mathcal{T}}\left(\underline{P}_{j t}^{d} \rho_{j t}^{l o}+\bar{P}_{j t}^{d} \rho_{j t}^{u p}+\sum_{b \in \mathcal{B}_{j}} \bar{P}_{b j t}^{d} \mu_{b j t}^{u p}\right)$

$+\sum_{i \in \mathcal{I}}\left[P_{i 0}^{g} \xi_{i 1}+R_{i}^{u p} V_{i 0} \xi_{i 1}+R_{i}^{s u}\left(v_{i 1} \xi_{i 1}-V_{i 0} \xi_{i 1}\right)\right.$

$$
\begin{gathered}
\left.+\bar{P}_{i 1}^{g}\left(\xi_{i 1}-v_{i 1} \xi_{i 1}\right)\right] \\
+\sum_{i \in \mathcal{I}} \sum_{t=2}^{n_{T}}\left[R_{i}^{u p} v_{i t-1} \xi_{i t}+R_{i}^{s u}\left(v_{i t} \xi_{i t}-v_{i t-1} \xi_{i t}\right)\right. \\
\left.\quad+\bar{P}_{i t}^{g}\left(\xi_{i t}-v_{i t} \xi_{i t}\right)\right] \\
+\sum_{i \in \mathcal{I}}\left[P_{i 0}^{g} \sigma_{i 1}-R_{i}^{d n} v_{i 1} \sigma_{i 1}-R_{i}^{s d}\left(V_{i 0} \sigma_{i 1}-v_{i 1} \sigma_{i 1}\right)\right. \\
\left.\quad-\bar{P}_{i 1}^{g}\left(1-V_{i 0}\right) \sigma_{i 1}\right]
\end{gathered}
$$

$$
\begin{gathered}
-\sum_{i \in \mathcal{I}} \sum_{t=2}^{n_{T}}\left[R_{i}^{d n} v_{i t} \sigma_{i t}+R_{i}^{s d}\left(v_{i t-1} \sigma_{i t}-v_{i t} \sigma_{i t}\right)\right. \\
\left.+\bar{P}_{i t}^{g}\left(\sigma_{i t}-v_{i t-1} \sigma_{i t}\right)\right] \\
+\sum_{i \in \mathcal{I}} \sum_{t=1}^{n_{T}-1}\left[R_{i}^{s d}\left(v_{i t} \epsilon_{i t}-v_{i t+1} \epsilon_{i t}\right)+\bar{P}_{i t}^{g} v_{i t+1} \epsilon_{i t}\right]
\end{gathered}
$$

where (25)-(27) correspond to the upper optimization level (1)-(8), whereas (28)-(42) equivalently replace the lower optimization level (9)-(21). The lower-level primal feasibility constraints are included in (28), expressions (29)-(41) represent the lower-level dual feasibility constraints, and (42) is the equality associated with the strong duality theorem.

\section{B. Step 2: Sorting Out Bilinear Terms}

Bilinear terms in the single-level equivalent (25)-(42) can be categorized as: 1) products of power outputs $p_{i t}^{g}$ and locational marginal prices $\lambda_{n t}$ in the definition of producer revenues (27), and 2) products of binary variables $v_{i t}$ and continuous dual variables in the strong duality equality (42).

As described next, bilinear products in (27) can be equivalently transformed into linear expressions based on KKT optimality conditions.

First, the summation of bilinear terms in the right-hand side of (27) can be expressed as follows:

$$
\sum_{t \in \mathcal{T}} p_{i t}^{g} \lambda_{n(i) t}=\sum_{t=1}^{n_{T}-1} p_{i t}^{g} \lambda_{n(i) t}+p_{i n_{T}}^{g} \lambda_{n(i) n_{T}} ; \forall i \in \mathcal{I} .
$$

Using (29) and (30), expression (43) becomes:

$$
\begin{aligned}
& \sum_{t \in \mathcal{T}} p_{i t}^{g} \lambda_{n(i) t}=\sum_{t \in \mathcal{T}}\left(p_{i t}^{g} \gamma_{i t}^{l o}+p_{i t}^{g} \gamma_{i t}^{u p}\right)+p_{i 1}^{g} \xi_{i 1} \\
& +\sum_{t=2}^{n_{T}}\left(p_{i t}^{g}-p_{i t-1}^{g}\right) \xi_{i t}+\sum_{t=1}^{n_{T}-1} p_{i t}^{g} \epsilon_{i t}+p_{i 1}^{g} \sigma_{i 1} \\
& +\sum_{t=2}^{n_{T}}\left(p_{i t}^{g}-p_{i t-1}^{g}\right) \sigma_{i t}+\sum_{t \in \mathcal{T}} p_{i t}^{g} \alpha_{i t} ; \forall i \in \mathcal{I} .
\end{aligned}
$$

Furthermore, the last summation in the right-hand side of (44) can be recast by using (15):

$$
\sum_{t \in \mathcal{T}} p_{i t}^{g} \alpha_{i t}=\sum_{o \in \mathcal{O}_{i}} \sum_{t \in \mathcal{T}} p_{o i t}^{g} \alpha_{i t} ; \forall i \in \mathcal{I}
$$

From (31), $\alpha_{i t}$ can be replaced in the right-hand side of (45) as follows:

$$
\sum_{t \in \mathcal{T}} p_{i t}^{g} \alpha_{i t}=\sum_{o \in \mathcal{O}_{i}} \sum_{t \in \mathcal{T}}\left(C_{\text {oit }}^{g} p_{\text {oit }}^{g}+p_{\text {oit }}^{g} \beta_{\text {oit }}^{\text {lo }}+p_{\text {oit }}^{g} \beta_{\text {oit }}^{u p}\right)
$$$$
\forall i \in \mathcal{I} \text {. }
$$ 
Therefore, using (46) in (44) yields:

$$
\begin{aligned}
& \sum_{t \in \mathcal{T}} p_{i t}^{g} \lambda_{n(i) t}=\sum_{t \in \mathcal{T}}\left(p_{i t}^{g} \gamma_{i t}^{l o}+p_{i t}^{g} \gamma_{i t}^{u p}\right)+p_{i 1}^{g} \xi_{i 1} \\
& +\sum_{t=2}^{n_{T}}\left(p_{i t}^{g}-p_{i t-1}^{g}\right) \xi_{i t}+\sum_{t=1}^{n_{T}-1} p_{i t}^{g} \epsilon_{i t}+p_{i 1}^{g} \sigma_{i 1} \\
& +\sum_{t=2}^{n_{T}}\left(p_{i t}^{g}-p_{i t-1}^{g}\right) \sigma_{i t}+\sum_{o \in \mathcal{O}_{i}} \sum_{t \in \mathcal{T}} C_{o i t}^{g} p_{\text {oit }}^{g} \\
& +\sum_{o \in \mathcal{O}_{i}} \sum_{t \in \mathcal{T}} p_{\text {oit }}^{g} \beta_{\text {oit }}^{l o}+\sum_{o \in \mathcal{O}_{i}} \sum_{t \in \mathcal{T}} p_{\text {oit }}^{g} \beta_{\text {oit }}^{u p} ; \forall i \in \mathcal{I} .
\end{aligned}
$$

In the right-hand side of (47) there are still terms involving products of continuous variables. Such bilinear terms can be sorted out by using the following complementary slackness conditions, which are associated with inequalities (13), (16), and (19)-(21) characterizing generation energy offers:

$$
\begin{aligned}
& \left(p_{i t}^{g}-\underline{P}_{i t}^{g} v_{i t}\right) \gamma_{i t}^{l o}=0 ; \forall i \in \mathcal{I}, \forall t \in \mathcal{T} \\
& \left(p_{i t}^{g}-\bar{P}_{i t}^{g} v_{i t}\right) \gamma_{i t}^{u p}=0 ; \forall i \in \mathcal{I}, \forall t \in \mathcal{T} \\
& \left(p_{o i t}^{g}-0\right) \beta_{o i t}^{l o}=0 ; \forall i \in \mathcal{I}, \forall o \in \mathcal{O}_{i}, \forall t \in \mathcal{T} \\
& \left(p_{o i t}^{g}-\bar{P}_{o i t}^{g}\right) \beta_{o i t}^{u p}=0 ; \forall i \in \mathcal{I}, \forall o \in \mathcal{O}_{i}, \forall t \in \mathcal{T} \\
& {\left[p_{i 1}^{g}-\left(P_{i 0}^{g}+R_{i}^{u p} V_{i 0}+R_{i}^{s u}\left(v_{i 1}-V_{i 0}\right)\right.\right.} \\
& \left.\left.+\bar{P}_{i 1}^{g}\left(1-v_{i 1}\right)\right)\right] \xi_{i 1}=0 ; \forall i \in \mathcal{I} \\
& {\left[p_{i t}^{g}-p_{i t-1}^{g}-\left(R_{i}^{u p} v_{i t-1}+R_{i}^{s u}\left(v_{i t}-v_{i t-1}\right)\right.\right.} \\
& \left.\left.+\bar{P}_{i t}^{g}\left(1-v_{i t}\right)\right)\right] \xi_{i t}=0 ; \forall i \in \mathcal{I}, \forall t=2 \ldots n_{T} \\
& {\left[p_{i 1}^{g}-\left(P_{i 0}^{g}-R_{i}^{d n} v_{i 1}-R_{i}^{s d}\left(V_{i 0}-v_{i 1}\right)\right.\right.} \\
& \left.\left.-\bar{P}_{i 1}^{g}\left(1-V_{i 0}\right)\right)\right] \sigma_{i 1}=0 ; \forall i \in \mathcal{I} \\
& {\left[p_{i t}^{g}-p_{i t-1}^{g}-\left(-R_{i}^{d n} v_{i t}-R_{i}^{s d}\left(v_{i t-1}-v_{i t}\right)\right.\right.} \\
& \left.\left.-\bar{P}_{i t}^{g}\left(1-v_{i t-1}\right)\right)\right] \sigma_{i t}=0 ; \forall i \in \mathcal{I}, \forall t=2 \ldots n_{T} \\
& {\left[p_{i t}^{g}-\left(R_{i}^{s d}\left(v_{i t}-v_{i t+1}\right)+\bar{P}_{i t}^{g} v_{i t+1}\right)\right] \epsilon_{i t}=0 ;} \\
& \forall i \in \mathcal{I}, \forall t=1 \ldots n_{T}-1 .
\end{aligned}
$$

From (48)-(56), the following equalities can be derived:

$$
\begin{aligned}
& \sum_{t \in \mathcal{T}} p_{i t}^{g} \gamma_{i t}^{l o}=\sum_{t \in \mathcal{T}} \underline{P}_{i t}^{g} v_{i t} \gamma_{i t}^{l o} ; \forall i \in \mathcal{I} \\
& \sum_{t \in \mathcal{T}} p_{i t}^{g} \gamma_{i t}^{u p}=\sum_{t \in \mathcal{T}} \bar{P}_{i t}^{g} v_{i t} \gamma_{i t}^{u p} ; \forall i \in \mathcal{I} \\
& \sum_{o \in \mathcal{O}_{i}} \sum_{t \in \mathcal{T}} p_{o i t}^{g} \beta_{o i t}^{l o}=0 ; \forall i \in \mathcal{I} \\
& \sum_{o \in \mathcal{O}_{i}} \sum_{t \in \mathcal{T}} p_{o i t}^{g} \beta_{o i t}^{u p}=\sum_{o \in \mathcal{O}_{i}} \sum_{t \in \mathcal{T}} \bar{P}_{o i t}^{g} \beta_{o i t}^{u p} ; \forall i \in \mathcal{I} \\
& p_{i 1}^{g} \xi_{i 1}=\left[P_{i 0}^{g}+R_{i}^{u p} V_{i 0}+R_{i}^{s u}\left(v_{i 1}-V_{i 0}\right)\right. \\
& \left.+\bar{P}_{i 1}^{g}\left(1-v_{i 1}\right)\right] \xi_{i 1} ; \forall i \in \mathcal{I}
\end{aligned}
$$

$$
\begin{aligned}
& \sum_{t=2}^{n_{T}}\left(p_{i t}^{g}-p_{i t-1}^{g}\right) \xi_{i t}=\sum_{t=2}^{n_{T}}\left[R_{i}^{u p} v_{i t-1}\right. \\
& \left.+R_{i}^{s u}\left(v_{i t}-v_{i t-1}\right)+\bar{P}_{i t}^{g}\left(1-v_{i t}\right)\right] \xi_{i t} ; \forall i \in \mathcal{I} \\
& p_{i 1}^{g} \sigma_{i 1}=\left[P_{i 0}^{g}-R_{i}^{d n} v_{i 1}-R_{i}^{s d}\left(V_{i 0}-v_{i 1}\right)\right. \\
& \left.-\bar{P}_{i 1}^{g}\left(1-V_{i 0}\right)\right] \sigma_{i 1} ; \forall i \in \mathcal{I} \\
& \sum_{t=2}^{n_{T}}\left(p_{i t}^{g}-p_{i t-1}^{g}\right) \sigma_{i t}=\sum_{t=2}^{n_{T}}\left[-R_{i}^{d n} v_{i t}\right. \\
& \left.-R_{i}^{s d}\left(v_{i t-1}-v_{i t}\right)-\bar{P}_{i t}^{g}\left(1-v_{i t-1}\right)\right] \sigma_{i t} ; \forall i \in \mathcal{I} \\
& \sum_{t=1}^{n_{T}-1} p_{i t}^{g} \epsilon_{i t}=\sum_{t=1}^{n_{T}-1}\left[R_{i}^{s d}\left(v_{i t}-v_{i t+1}\right)\right. \\
& \left.+\bar{P}_{i t}^{g} v_{i t+1}\right] \epsilon_{i t} ; \forall i \in \mathcal{I} .
\end{aligned}
$$

Note that the bilinear terms in the right-hand side of (47) correspond to the left-hand sides of (57)-(65). Thus, replacing them in (47) results in:

$$
\begin{aligned}
& \sum_{t \in \mathcal{T}} p_{i t}^{g} \lambda_{n(i) t}=\sum_{t \in \mathcal{T}}\left(\underline{P}_{i t}^{g} v_{i t} \gamma_{i t}^{l o}+\bar{P}_{i t}^{g} v_{i t} \gamma_{i t}^{u p}\right) \\
& +\left[P_{i 0}^{g} \xi_{i 1}+R_{i}^{u p} V_{i 0} \xi_{i 1}+R_{i}^{s u}\left(v_{i 1} \xi_{i 1}-V_{i 0} \xi_{i 1}\right)\right. \\
& \left.\quad+\bar{P}_{i 1}^{g}\left(\xi_{i 1}-v_{i 1} \xi_{i 1}\right)\right] \\
& +\sum_{t=2}^{n_{T}}\left[R_{i}^{u p} v_{i t-1} \xi_{i t}+R_{i}^{s u}\left(v_{i t} \xi_{i t}-v_{i t-1} \xi_{i t}\right)\right. \\
& \left.\quad+\bar{P}_{i t}^{g}\left(\xi_{i t}-v_{i t} \xi_{i t}\right)\right] \\
& +\left[P_{i 0}^{g} \sigma_{i 1}-R_{i}^{d n} v_{i 1} \sigma_{i 1}-R_{i}^{s d}\left(V_{i 0} \sigma_{i 1}-v_{i 1} \sigma_{i 1}\right)\right. \\
& \left.\quad-\bar{P}_{i 1}^{g}\left(1-V_{i 0}\right) \sigma_{i 1}\right] \\
& -\sum_{t=2}^{n_{T}}\left[R_{i}^{d n} v_{i t} \sigma_{i t}+R_{i}^{s d}\left(v_{i t-1} \sigma_{i t}-v_{i t} \sigma_{i t}\right)\right. \\
& \left.\quad+\bar{P}_{i t}^{g}\left(\sigma_{i t}-v_{i t-1} \sigma_{i t}\right)\right] \\
& +\sum_{t=1}^{n_{T}-1}\left[R_{i}^{s d}\left(v_{i t} \epsilon_{i t}-v_{i t+1} \epsilon_{i t}\right)+\bar{P}_{i t}^{g} v_{i t+1} \epsilon_{i t}\right] \\
& +\sum_{o \in \mathcal{O}_{i}} \sum_{t \in \mathcal{T}} p_{o i t}^{g} C_{o i t}^{g}+\sum_{o \in \mathcal{O}_{i}} \sum_{t \in \mathcal{T}} \bar{P}_{o i t}^{g} \beta_{o i t}^{u p} ; \forall i \in \mathcal{I} .
\end{aligned}
$$

As a consequence, the bilinear terms involving products of continuous decision variables are expressed as sums of terms including products of binary decision variables and continuous dual variables. Such products are similar to those in the strong duality equality (42) resulting from the primaldual transformation. Using previously reported integer algebra results [47], such bilinear terms can be equivalently recast as:

$$
\begin{aligned}
& \underline{\gamma}_{i t}^{l o} v_{i t} \leq a_{i t} \leq 0 ; \forall i \in \mathcal{I}, \forall t \in \mathcal{T} \\
& \underline{\gamma}_{i t}^{l o}\left(1-v_{i t}\right) \leq \gamma_{i t}^{l o}-a_{i t} \leq 0 ; \forall i \in \mathcal{I}, \forall t \in \mathcal{T} \\
& 0 \leq b_{i t} \leq \bar{\gamma}_{i t}^{u p} v_{i t} ; \forall i \in \mathcal{I}, \forall t \in \mathcal{T} \\
& 0 \leq \gamma_{i t}^{u p}-b_{i t} \leq \bar{\gamma}_{i t}^{u p}\left(1-v_{i t}\right) ; \forall i \in \mathcal{I}, \forall t \in \mathcal{T} \\
& 0 \leq c_{i t} \leq \bar{\xi}_{i t} v_{i t} ; \forall i \in \mathcal{I}, \forall t \in \mathcal{T}
\end{aligned}
$$




$$
\begin{aligned}
& 0 \leq \xi_{i t}-c_{i t} \leq \bar{\xi}_{i t}\left(1-v_{i t}\right) ; \forall i \in \mathcal{I}, \forall t \in \mathcal{T} \\
& 0 \leq d_{i t} \leq \bar{\xi}_{i t} v_{i t-1} ; \forall i \in \mathcal{I}, \forall t=2 \ldots n_{T} \\
& 0 \leq \xi_{i t}-d_{i t} \leq \bar{\xi}_{i t}\left(1-v_{i t-1}\right) ; \forall i \in \mathcal{I}, \forall t=2 \ldots n_{T} \\
& 0 \leq e_{i t} \leq \bar{\epsilon}_{i t} v_{i t} ; \forall i \in \mathcal{I}, \forall t=1 \ldots n_{T}-1 \\
& 0 \leq \epsilon_{i t}-e_{i t} \leq \bar{\epsilon}_{i t}\left(1-v_{i t}\right) ; \forall i \in \mathcal{I}, \forall t=1 \ldots n_{T}-1 \\
& 0 \leq k_{i t} \leq \bar{\epsilon}_{i t} v_{i t+1} ; \forall i \in \mathcal{I}, \forall t=1 \ldots n_{T}-1 \\
& 0 \leq \epsilon_{i t}-k_{i t} \leq \bar{\epsilon}_{i t}\left(1-v_{i t+1}\right) ; \forall i \in \mathcal{I}, \forall t=1 \ldots n_{T}-1 \\
& \underline{\sigma}_{i t} v_{i t} \leq q_{i t} \leq 0 ; \forall i \in \mathcal{I}, \forall t \in \mathcal{T} \\
& \underline{\sigma}_{i t}\left(1-v_{i t}\right) \leq \sigma_{i t}-q_{i t} \leq 0 ; \forall i \in \mathcal{I}, \forall t \in \mathcal{T} \\
& \underline{\sigma}_{i t} v_{i t-1} \leq w_{i t} \leq 0 ; \forall i \in \mathcal{I}, \forall t=2 \ldots n_{T} \\
& \underline{\sigma}_{i t}\left(1-v_{i t-1}\right) \leq \sigma_{i t}-w_{i t} \leq 0 ; \forall i \in \mathcal{I}, \forall t=2 \ldots n_{T}
\end{aligned}
$$

where $a_{i t}, b_{i t}, c_{i t}, d_{i t}, e_{i t}, k_{i t}, q_{i t}$, and $w_{i t}$ represent $v_{i t} \gamma_{i t}^{l o}$, $v_{i t} \gamma_{i t}^{u p}, v_{i t} \xi_{i t}, v_{i t-1} \xi_{i t}, v_{i t} \epsilon_{i t}, v_{i t+1} \epsilon_{i t}, v_{i t} \sigma_{i t}$, and $v_{i t-1} \sigma_{i t}$, respectively. Expressions (67)-(68), (69)-(70), (71)-(72), (73)(74), (75)-(76), (77)-(78), (79)-(80), and (81)-(82) model the respective linearizations.

It should be noted that the above linearizations require setting bounds on lower-level dual variables $\gamma_{i t}^{l o}, \gamma_{i t}^{u p}, \epsilon_{i t}, \xi_{i t}$, and $\sigma_{i t}$. As described in [37], these bounding parameters may be selected based on the values of the corresponding dual variables resulting from the optimal solution to the associated revenue-unconstrained auction model.

\section{Single-Level Mixed-Integer Linear Equivalent}

The single-level equivalent of problem (1)-(21) is formulated as the following mixed-integer linear program:

$$
\begin{aligned}
& \underset{a_{i t}, b_{i t}, c_{i t}, d_{i t}, e_{i t},}{\operatorname{Maximize}} \quad \sum_{j \in \mathcal{J}} \sum_{b \in \mathcal{B}_{j}} \sum_{t \in \mathcal{T}} C_{b j t}^{d} p_{b j t}^{d} \\
& k_{i t}, p_{b j t}^{d}, p_{j t}^{d}, p_{l t}^{f}, p_{i t}^{g} \text {, } \\
& p_{o i t}^{g}, q_{i t}, r_{i}, s_{i t}^{s d}, s_{i t}^{s u} \text {, } \\
& v_{i t}, w_{i t}, z_{i}, \alpha_{i t}, \beta_{o i t}^{\text {lo }} \text {, } \\
& \beta_{o i t}^{u p}, \gamma_{i t}^{l o}, \gamma_{i t}^{u p}, \delta_{n t}, \epsilon_{i t}, \\
& \theta_{j t}, \lambda_{n t}, \mu_{b j t}^{l o}, \mu_{b j t}^{u p} \text {, } \\
& \nu_{l t}, \xi_{i t}, \rho_{j t}^{l o}, \rho_{j t}^{u p} \text {, } \\
& \sigma_{i t}, \phi_{l t}^{l o}, \phi_{l t}^{u p} \\
& -\sum_{i \in \mathcal{I}} \sum_{t \in \mathcal{T}}\left(\sum_{o \in \mathcal{O}_{i}} C_{o i t}^{g} p_{o i t}^{g}+s_{i t}^{s d}+s_{i t}^{s u}\right. \\
& \left.+C_{i t}^{n l} v_{i t}\right)
\end{aligned}
$$

subject to:

Constraints (2)-(6) and (8)

$$
r_{i}=\sum_{t \in \mathcal{T}}\left(\underline{P}_{i t}^{g} a_{i t}+\bar{P}_{i t}^{g} b_{i t}\right)
$$$$
+\left[P_{i 0}^{g} \xi_{i 1}+R_{i}^{u p} V_{i 0} \xi_{i 1}+R_{i}^{s u}\left(c_{i 1}-V_{i 0} \xi_{i 1}\right)\right.
$$$$
\left.+\bar{P}_{i 1}^{g}\left(\xi_{i 1}-c_{i 1}\right)\right]
$$$$
+\sum_{t=2}^{n_{T}}\left[R_{i}^{u p} d_{i t}+R_{i}^{s u}\left(c_{i t}-d_{i t}\right)+\bar{P}_{i t}^{g}\left(\xi_{i t}-c_{i t}\right)\right]
$$$$
+\left[P_{i 0}^{g} \sigma_{i 1}-R_{i}^{d n} q_{i 1}-R_{i}^{s d}\left(V_{i 0} \sigma_{i 1}-q_{i 1}\right)\right.
$$$$
\left.-\bar{P}_{i 1}^{g}\left(1-V_{i 0}\right) \sigma_{i 1}\right]
$$$$
-\sum_{t=2}^{n_{T}}\left[R_{i}^{d n} q_{i t}+R_{i}^{s d}\left(w_{i t}-q_{i t}\right)+\bar{P}_{i t}^{g}\left(\sigma_{i t}-w_{i t}\right)\right]
$$

TABLE I

DiMENSION OF PROBLEM (83)-(89)

\begin{tabular}{crr}
\hline \# of constraints & $\begin{array}{c}\text { \# of binary } \\
\text { variables }\end{array}$ & $\begin{array}{r}\text { \# of rea } \\
\text { variables }\end{array}$ \\
\hline $2 n_{T}\left(n_{N}+2 n_{L}+2 n_{J}\right)$ & & $2 n_{T}\left(n_{N}+2 n_{L}\right.$ \\
+ & $n_{I}\left(44 n_{T}-10\right)$ & $+n_{I}\left(17 n_{T}\right.$ \\
$+3 n_{T} \sum_{i \in \mathcal{I}} n_{O_{i}}$ & $n_{I} n_{T}$ & $+3 n_{T} \sum_{i \in \mathcal{I}}$ \\
$+3 n_{T} \sum_{j \in \mathcal{J}} n_{B_{j}}+2$ & $+3 n_{T} \sum_{j \in \mathcal{J}}$ \\
& & \\
$+\sum_{t=1}^{n_{T}-1}\left[R_{i}^{s d}\left(e_{i t}-k_{i t}\right)+\bar{P}_{i t}^{g} k_{i t}\right]$ & \\
$+\sum_{o \in \mathcal{O}_{i}} \sum_{t \in \mathcal{T}} p_{\text {oit }}^{g} C_{\text {oit }}^{g}+\sum_{o \in \mathcal{O}_{i}} \sum_{t \in \mathcal{T}} \bar{P}_{\text {oit }}^{g} \beta_{\text {oit }}^{\text {up }} ;$ &
\end{tabular}

Constraints (10)-(21)

Constraints (29)-(41)

$$
\begin{aligned}
& \sum_{j \in \mathcal{J}} \sum_{b \in \mathcal{B}_{j}} \sum_{t \in \mathcal{T}} C_{b j t}^{d} p_{b j t}^{d}-\sum_{i \in \mathcal{I}} \sum_{o \in \mathcal{O}_{i}} \sum_{t \in \mathcal{T}} C_{o i t}^{g} p_{o i t}^{g}= \\
& \sum_{l \in \mathcal{L}} \sum_{t \in \mathcal{T}} \bar{P}_{l}^{f}\left(\phi_{l t}^{u p}-\phi_{l t}^{l o}\right) \\
& +\sum_{i \in \mathcal{I}} \sum_{t \in \mathcal{T}}\left(\underline{P}_{i t}^{g} a_{i t}+\bar{P}_{i t}^{g} b_{i t}+\sum_{o \in \mathcal{O}_{i}} \bar{P}_{o i t}^{g} \beta_{o i t}^{u p}\right) \\
& +\sum_{j \in \mathcal{J}} \sum_{t \in \mathcal{T}}\left(\underline{P}_{j t}^{d} \rho_{j t}^{l o}+\bar{P}_{j t}^{d} \rho_{j t}^{u p}+\sum_{b \in \mathcal{B}_{j}} \bar{P}_{b j t}^{d} \mu_{b j t}^{u p}\right) \\
& +\sum_{i \in \mathcal{I}}\left[P_{i 0}^{g} \xi_{i 1}+R_{i}^{u p} V_{i 0} \xi_{i 1}+R_{i}^{s u}\left(c_{i 1}-V_{i 0} \xi_{i 1}\right)\right. \\
& \left.+\bar{P}_{i 1}^{g}\left(\xi_{i 1}-c_{i 1}\right)\right] \\
& +\sum_{i \in \mathcal{I}} \sum_{t=2}^{n_{T}}\left[R_{i}^{u p} d_{i t}+R_{i}^{s u}\left(c_{i t}-d_{i t}\right)+\bar{P}_{i t}^{g}\left(\xi_{i t}-c_{i t}\right)\right] \\
& +\sum_{i \in \mathcal{I}}\left[P_{i 0}^{g} \sigma_{i 1}-R_{i}^{d n} q_{i 1}-R_{i}^{s d}\left(V_{i 0} \sigma_{i 1}-q_{i 1}\right)\right. \\
& \left.\quad-\bar{P}_{i 1}^{g}\left(1-V_{i 0}\right) \sigma_{i 1}\right] \\
& +\sum_{i \in \mathcal{I}} \sum_{t=2}^{n_{T}}\left[R_{i}^{d n} q_{i t}+R_{i}^{s d}\left(w_{i t}-q_{i t}\right)+\bar{P}_{i t}^{g}\left(\sigma_{i t}-w_{i t}\right)\right] \\
& +\sum_{i \in \mathcal{I}} \sum_{t=1}^{n_{T}-1}\left[R_{i}^{s d}\left(e_{i t}-k_{i t}\right)+\bar{P}_{i t}^{g} k_{i t}\right] \\
& C o i n
\end{aligned}
$$

Constraints (67)-(82)

where (83)-(84) correspond to (1)-(6) and (8), expressions (85) are the linear revenue terms, expressions (86)-(87) respectively include the lower-level primal and dual feasibility constraints, expression (88) is the linear equality associated with the strong duality theorem, and (89) models the linearization of the products of upper-level binary decision variables and lower-level continuous dual variables.

Table I provides the size of the single-level mixed-integer linear equivalent (83)-(89) in terms of the numbers of constraints, binary variables, and real variables. It is worth em- 
phasizing that the number of minimum up and down time constraints depends on the initial statuses of generators. Therefore, the number of constraints listed in Table I is an upper bound on the corresponding actual number of constraints.

\section{Conclusion}

This paper has presented an exact bilevel-programmingbased approach to address revenue- and network-constrained market clearing within the context of pool-based day-ahead electricity markets under marginal pricing. For over a decade, this auction model, which constitutes the solution adopted in national electricity markets across Europe to ensure revenue sufficiency, has been routinely albeit inadequately solved by European system operators. The revenue-constrained auction design is a particular instance of price-based market clearing wherein locational marginal prices become decision variables of the optimization. Moreover, revenue terms are formulated as bilinear and hence highly nonconvex products of power outputs and locational marginal prices. Practical modeling aspects allow converting the resulting mixed-integer nonlinear bilevel program with bilinear terms into a single-level mixedinteger linear equivalent with no extra binary variables. This transformation applies results from duality theory of linear programming, Karush-Kuhn-Tucker optimality conditions, and integer algebra.

The proposed model and solution methodology feature relevant aspects for the purposes of practical implementation: 1) the resulting generation and consumption levels are consistent with an economic dispatch problem, 2) the resulting vector of locational market-clearing prices complies with marginal pricing, 3) price uplifts are not required, and 4) both minimum revenue conditions and minimum declared profits can be handled.

The second part of this two-paper series provides a detailed analysis of three case studies, namely 1) an illustrative five-bus example, 2) a case based on the IEEE Reliability Test System, and 3) a case based on the IEEE 118-bus system. Results from these case studies are compared with those achieved by available techniques in order to show the effectiveness of the proposed methodology. In addition, computational issues are discussed on the basis of the computing times required to attain high-quality solutions for a suite of benchmarks of increasing size including a case study based on the IEEE-300 bus system.

\section{APPENDIX}

The feasibility set $\mathcal{F}_{i t}$ for variables $s_{i t}^{s d}, s_{i t}^{s u}$, and $v_{i t}$ is characterized by the following set of linear constraints:

$$
\begin{aligned}
& \sum_{t=1}^{n_{i}^{U T}}\left(1-v_{i t}\right)=0 ; \forall i \in \mathcal{I} \\
& \sum_{h=t}^{t+U T_{i}-1} v_{i h} \geq U T_{i}\left(v_{i t}-v_{i t-1}\right) \\
& \forall i \in \mathcal{I}, \forall t=n_{i}^{U T}+1 \ldots n_{T}-U T_{i}+1
\end{aligned}
$$

$$
\begin{aligned}
& \sum_{h=t}^{n_{T}}\left[v_{i h}-\left(v_{i t}-v_{i t-1}\right)\right] \geq 0 ; \\
& \forall i \in \mathcal{I}, \forall t=n_{T}-U T_{i}+2 \ldots n_{T} \\
& \sum_{t=1}^{n_{i}^{D T}} v_{i t}=0 ; \forall i \in \mathcal{I} \\
& \sum_{h=t}^{t+D T_{i}-1}\left(1-v_{i h}\right) \geq D T_{i}\left(v_{i t-1}-v_{i t}\right) ; \\
& \forall i \in \mathcal{I}, \forall t=n_{i}^{D T}+1 \ldots n_{T}-D T_{i}+1 \\
& \sum_{h=t}^{n_{T}}\left[1-v_{i h}-\left(v_{i t-1}-v_{i t}\right)\right] \geq 0 \text {; } \\
& \forall i \in \mathcal{I}, \forall t=n_{T}-D T_{i}+2 \ldots n_{T} \\
& s_{i t}^{s d} \geq C_{i t}^{s d}\left(v_{i t-1}-v_{i t}\right) ; \forall i \in \mathcal{I}, \forall t \in \mathcal{T} \\
& s_{i t}^{s d} \geq 0 ; \forall i \in \mathcal{I}, \forall t \in \mathcal{T} \\
& s_{i t}^{s u} \geq C_{i t}^{s u}\left(v_{i t}-v_{i t-1}\right) ; \forall i \in \mathcal{I}, \forall t \in \mathcal{T} \\
& s_{i t}^{s u} \geq 0 ; \forall i \in \mathcal{I}, \forall t \in \mathcal{T}
\end{aligned}
$$

where $n_{i}^{D T}=\min \left\{n_{T},\left(D T_{i}-n_{i 0}^{o f f}\right)\left(1-V_{i 0}\right)\right\}$ and $n_{i}^{U T}=\min \left\{n_{T},\left(U T_{i}-n_{i 0}^{o n}\right) V_{i 0}\right\}$.

Minimum up and down time constraints are respectively modeled in (90)-(92) and (93)-(95), whereas (96)-(97) and (98)-(99) respectively correspond to shut-down and start-up offers. The interested reader is referred to [45] for a detailed description of these linear constraints.

\section{REFERENCES}

[1] R. Fernández-Blanco, J. M. Arroyo, and N. Alguacil, "On the solution of revenue- and network-constrained day-ahead market clearing under marginal pricing-Part II: Case studies," submitted to IEEE Trans. Power Syst.

[2] R. Baldick, U. Helman, B. F. Hobbs, and R. P. O'Neill, "Design of efficient generation markets," Proc. IEEE, vol. 93, no. 11, pp. 1998 2012, Nov. 2005.

[3] F. P. Sioshansi, Competitive Electricity Markets: Design, Implementation, Performance. Amsterdam, The Netherlands: Elsevier, 2008.

[4] M. Shahidehpour, H. Yamin, and Z. Li, Market Operations in Electric Power Systems: Forecasting, Scheduling, and Risk Management. New York, NY, USA: John Wiley \& Sons, Inc., 2002.

[5] A. Gómez-Expósito, A. J. Conejo, and C. Cañizares, Electric Energy Systems: Analysis and Operation. Boca Raton, FL, USA: CRC Press, 2008.

[6] R. B. Johnson, S. S. Oren, and A. J. Svoboda, "Equity and efficiency of unit commitment in competitive electricity markets," Util. Policy, vol. 6, no. 1, pp. 9-19, Mar. 1997.

[7] PJM, "A review of generation compensation and cost elements in the PJM markets," 2009. [Online]. Available: http://www. pjm.com/ /media/committees-groups/committees/mrc/20100120/ 20100120-item-02-review-of-generation-costs-and-compensation.ashx

[8] ISO New England, "FAQs: Net commitment-period compensation (NCPC)," 2016. [Online]. Available: http://www.iso-ne.com/participate/ support/faq/ncpc-rmr

[9] A. L. Motto and F. D. Galiana, "Equilibrium of auction markets with unit commitment: The need for augmented pricing," IEEE Trans. Power Syst., vol. 17, no. 3, pp. 798-805, Aug. 2002.

[10] F. D. Galiana, A. L. Motto, and F. Bouffard, "Reconciling social welfare, agent profits, and consumer payments in electricity pools," IEEE Trans. Power Syst., vol. 18, no. 2, pp. 452-459, May 2003.

[11] S. Hao and F. Zhuang, "New models for integrated short-term forward electricity markets," IEEE Trans. Power Syst., vol. 18, no. 2, pp. 478485, May 2003. 
[12] R. P. O’Neill, P. M. Sotkiewicz, B. F. Hobbs, M. H. Rothkopf, and W. R. Stewart, Jr., "Efficient market-clearing prices in markets with nonconvexities," Eur. J. Oper. Res., vol. 164, no. 1, pp. 269-285, Jul. 2005.

[13] F. Bouffard and F. D. Galiana, "Generalized uplifts in pool-based electricity markets," in Analysis, Control and Optimization of Complex Dynamic Systems, E. K. Boukas and R. P. Malhamé, Eds. New York, NY, USA: Springer, 2005, pp. 193-214.

[14] M. Bjørndal and K. Jörnsten, "Equilibrium prices supported by dual price functions in markets with non-convexities," Eur. J. Oper. Res., vol. 190 , no. 3, pp. 768-789, Nov. 2008.

[15] B. Zhang, P. B. Luh, E. Litvinov, T. Zheng, and F. Zhao, "On reducing uplift payment in electricity markets," presented at the IEEE PES Power Syst. Conf. Exposition, Seattle, WA, USA, Mar. 2009.

[16] C. Ruiz, A. J. Conejo, and S. A. Gabriel, "Pricing non-convexities in an electricity pool," IEEE Trans. Power Syst., vol. 27, no. 3, pp. 1334-1342, Aug. 2012.

[17] S. A. Gabriel, A. J. Conejo, C. Ruiz, and S. Siddiqui, "Solving discretely-constrained, mixed linear complementarity problems with applications in energy," Comput. Oper. Res., vol. 40, no. 5, pp. 1339 1350, May 2013.

[18] P. Andrianesis, G. Liberopoulos, G. Kozanidis, and A. D. Papalexopoulos, "Recovery mechanisms in day-ahead electricity markets with nonconvexities-Part I: Design and evaluation methodology," IEEE Trans. Power Syst., vol. 28, no. 2, pp. 960-968, May 2013.

[19] _ - "Recovery mechanisms in day-ahead electricity markets with nonconvexities-Part II: Implementation and numerical evaluation," IEEE Trans. Power Syst., vol. 28, no. 2, pp. 969-977, May 2013.

[20] Market Operator of the Iberian Electricity Market, "Daily and intraday electricity market operating rules," 2014. [Online]. Available: http://www.omel.es/files/20140509_reglas_v11_ingles.pdf

[21] Power Spot Exchange NL, 2016. [Online]. Available: http://www. apxgroup.com/trading-clearing/day-ahead-auction/

[22] S. Hao, G. A. Angelidis, H. Singh, and A. D. Papalexopoulos, "Consumer payment minimization in power pool auctions," IEEE Trans. Power Syst., vol. 13, no. 3, pp. 986-991, Aug. 1998.

[23] J. Alonso, A. Trías, V. Gaitan, and J. J. Alba, "Thermal plant bids and market clearing in an electricity pool. Minimization of costs vs. minimization of consumer payments," IEEE Trans. Power Syst., vol. 14, no. 4, pp. 1327-1334, Nov. 1999.

[24] C. Vázquez, M. Rivier, and I. J. Pérez-Arriaga, "Production cost minimization versus consumer payment minimization in electricity pools," IEEE Trans. Power Syst., vol. 17, no. 1, pp. 119-127, Feb. 2002.

[25] Y. Ren and F. D. Galiana, "Minimum consumer payment scheduling and pricing in electricity markets," presented at the 14th Power Syst. Comput. Conf., PSCC'02, Seville, Spain, Jun. 2002.

[26] A. L. Motto and F. D. Galiana, "Coordination in markets with nonconvexities as a mathematical program with equilibrium constraints-Part I: A solution procedure," IEEE Trans. Power Syst., vol. 19, no. 1, pp. 308-316, Feb. 2004.

[27] _ - "Coordination in markets with nonconvexities as a mathematical program with equilibrium constraints-Part II: Case studies," IEEE Trans. Power Syst., vol. 19, no. 1, pp. 317-324, Feb. 2004

[28] — - "Unit commitment with dual variable constraints," IEEE Trans. Power Syst., vol. 19, no. 1, pp. 330-338, Feb. 2004.

[29] R. García-Bertrand, A. J. Conejo, and S. A. Gabriel, "Multi-period near-equilibrium in a pool-based electricity market including on/off decisions," Netw. Spat. Econ., vol. 5, no. 4, pp. 371-393, Dec. 2005.

[30] R. García-Bertrand, A. J. Conejo, and S. Gabriel, "Electricity market near-equilibrium under locational marginal pricing and minimum profit conditions," Eur. J. Oper. Res., vol. 174, no. 1, pp. 457-479, Oct. 2006.

[31] P. B. Luh, Y. Chen, J. H. Yan, G. A. Stern, W. E. Blankson, and F. Zhao, "Payment cost minimization with demand bids and partial capacity cost compensations for day-ahead electricity auctions," in Economic Market Design and Planning for Electric Power Systems, J. Momoh and L. Mili, Eds. New Jersey, NJ, USA: John Wiley \& Sons, Inc., 2010, pp. 71-85.

[32] EPEX Spot, APX, Belpex, Nord Pool Spot, OMIE, Mercatoelettrico (GME), and OTE, "EUPHEMIA public description: PCR market coupling algorithm," Oct. 2013. [Online]. Available: http://nordpoolspot.com/globalassets/download-center/pcr/euphemiapublic-description_nov2013.pdf

[33] European Commission, "A fully-integrated internal energy market," 2016. [Online]. Available: http://ec.europa.eu/priorities/energy-unionand-climate/fully-integrated-internal-energy-market_en

[34] C.-A. Li, A. J. Svoboda, X. Guan, and H. Singh, "Revenue adequate bidding strategies in competitive electricity markets," IEEE Trans. Power Syst., vol. 14, no. 2, pp. 492-497, May 1999.
[35] F. C. Schweppe, M. C. Caramanis, R. D. Tabors, and R. E. Bohn, Spot Pricing of Electricity. Norwell, MA, USA: Kluwer Academic Publishers, 1988.

[36] A. L. Motto, F. D. Galiana, A. J. Conejo, and J. M. Arroyo, "Networkconstrained multiperiod auction for a pool-based electricity market," IEEE Trans. Power Syst., vol. 17, no. 3, pp. 646-653, Aug. 2002.

[37] R. Fernández-Blanco, J. M. Arroyo, and N. Alguacil, "A unified bilevel programming framework for price-based market clearing under marginal pricing," IEEE Trans. Power Syst., vol. 27, no. 1, pp. 517-525, Feb. 2012.

[38] Z.-Q. Luo, J.-S. Pang, and D. Ralph, Mathematical Programs with Equilibrium Constraints. Cambridge, UK: Cambridge University Press, 1996.

[39] R. A. Horn and C. R. Johnson, Matrix Analysis, 2nd ed. New York, NY, USA: Cambridge University Press, 2012.

[40] M. V. Pereira, S. Granville, M. H. C. Fampa, R. Dix, and L. A. Barroso, "Strategic bidding under uncertainty: A binary expansion approach," IEEE Trans. Power Syst., vol. 20, no. 1, pp. 180-188, Feb. 2005.

[41] S. Dempe, Foundations of Bilevel Programming. Dordrecht, The Netherlands: Kluwer Academic Publishers, 2002.

[42] J. M. Arroyo, "Bilevel programming applied to power system vulnerability analysis under multiple contingencies," IET Gener. Transm. Distrib., vol. 4, no. 2, pp. 178-190, Feb. 2010.

[43] G. L. Nemhauser and L. A. Wolsey, Integer and Combinatorial Optimization. New York, NY, USA: Wiley-Interscience, 1999.

[44] The IBM ILOG CPLEX website, 2016. [Online]. Available: http://www01.ibm.com/software/commerce/optimization/cplex-optimizer

[45] M. Carrión and J. M. Arroyo, "A computationally efficient mixed-integer linear formulation for the thermal unit commitment problem," IEEE Trans. Power Syst., vol. 21, no. 3, pp. 1371-1378, Aug. 2006.

[46] D. G. Luenberger, Linear and Nonlinear Programming, 2nd ed. Reading, MA, USA: Addison-Wesley, 1989.

[47] C. A. Floudas, Nonlinear and Mixed-Integer Optimization: Fundamentals and Applications. New York, NY, USA: Oxford University Press, 1995.

Ricardo Fernández-Blanco (S'10-M'15) received the Ingeniero Industrial degree and the Ph.D. degree in electrical engineering from the Universidad de Castilla-La Mancha, Ciudad Real, Spain, in 2009 and 2014, respectively.

$\mathrm{He}$ is currently a postdoctoral researcher at University of Washington, Seattle, WA, USA. His research interests are in the fields of energy and power systems, bilevel programming, and electricity markets.

José M. Arroyo (S'96-M'01-SM'06) received the Ingeniero Industrial degree from the Universidad de Málaga, Málaga, Spain, in 1995, and the Ph.D. degree in power systems operations planning from the Universidad de Castilla-La Mancha, Ciudad Real, Spain, in 2000.

From June 2003 through July 2004 he held a Richard H. Tomlinson Postdoctoral Fellowship at the Department of Electrical and Computer Engineering of McGill University, Montreal, QC, Canada. He is currently a Full Professor of electrical engineering at the Universidad de Castilla-La Mancha. His research interests include operations, planning, and economics of power systems, as well as optimization.

Natalia Alguacil (S'97-M'01-SM'07) received the Ingeniero en Informática degree from the Universidad de Málaga, Málaga, Spain, in 1995, and the $\mathrm{Ph} . \mathrm{D}$. degree in power systems operations and planning from the Universidad de Castilla-La Mancha, Ciudad Real, Spain, in 2001.

She is currently an Associate Professor of electrical engineering at the Universidad de Castilla-La Mancha. Her research interests include operations, planning, and economics of power systems. 